\title{
Xinmailong Injection for Improvement of Cardiac Function in Patients with Heart Failure: A Systematic Review and Meta-Analysis
}

\author{
Yuan-long Sun, ${ }^{1,2}$ Yi-ping Li, ${ }^{1,2}$ Ting-ting Qiang, ${ }^{1,2}$ Xiao-fen Ruan $\mathbb{D}^{1,2}$ \\ and Xiao-long Wang $\mathbb{B}^{1,2}$ \\ ${ }^{1}$ Cardiovascular Department, Shuguang Hospital of Shanghai University of Traditional Chinese Medicine, \\ Shanghai 201203, China \\ ${ }^{2}$ Cardiovascular Research Institute of Traditional Chinese Medicine, \\ Shuguang Hospital of Shanghai University of Traditional Chinese Medicine, Shanghai 201203, China
}

Correspondence should be addressed to Xiao-fen Ruan; ruanxiaofeng@shutcm.edu.cn and Xiao-long Wang; wangxiaolong@ shutcm.edu.cn

Received 28 June 2020; Revised 16 October 2020; Accepted 28 October 2020; Published 19 November 2020

Academic Editor: Sai-Wang Seto

Copyright (c) 2020 Yuan-long Sun et al. This is an open access article distributed under the Creative Commons Attribution License, which permits unrestricted use, distribution, and reproduction in any medium, provided the original work is properly cited.

\begin{abstract}
Background. Insect drugs have great potential for treating cardiovascular diseases. Xinmailong (XML) injection, a bioactive composite extracted from Periplaneta americana (a species of cockroach), was wildly used in treating heart failure in China. This meta-analysis aimed to assess the efficacy and safety of XML injection for the improvement of cardiac function in HF. Materials and Methods. Online literature search for relevant studies was performed using databases including PubMed, EMBASE, Cochrane Library, CNKI, and Wanfang. Left ventricular ejection fraction (LVEF), six-minute walk test (6MWT), and brain natriuretic peptide (BNP) were selected as target outcomes. The analysis was performed using Stata 12.0, and sources of heterogeneity were explored by subgroup analysis and metaregression. Results. 32 studies were included in this meta-analysis after meeting the inclusion/exclusion criteria. The results demonstrated that additional use of XML improved LVEF (WMD = 5.82, 95\% CI: 5.52-7.13, $P<0.00001)$ and $6 \mathrm{MWT}(\mathrm{WMD}=51.48$, 95\% CI: 35.83-67.13, $P<0.00001)$ and reduced BNP (WMD $=-172.84,95 \%$ CI: -205.79 to $-139.89, P<0.00001$ ). The results of subgroup analyses and metaregression suggested that XML injection has more cardiac function improvement for middle-aged HF patients than youth, and greater LVEF and 6MWT improvement were associated with higher average age. Conclusions. XML plus conventional treatment demonstrated a significant effect in reducing cardiac dysfunction in HF patients, and age is a potential factor of higher efficacy. Given the heterogeneity and bias of the included RCTs, large, prospective, rigorous trials are still needed.
\end{abstract}

\section{Introduction}

Heart failure (HF) is a global healthcare issue, defined as a severe and terminal-stage symptom after heart disease, and has high patient mortality $[1,2]$. HF induces structural, neurohumoral, cellular, and molecular dysfunctions and leads to several organ and system dysfunction resulting in complex clinical manifestations [3]. About $12 \%$ of individuals over 80 have HF [4]. As of $2015,8.9 \%$ of Chinese population over 35 years of age have HF [5].

Insect drugs, a type of traditional Chinese medicine (TCM), have great potential for the treatment of several cardiovascular diseases. Insect extracts are rich sources of pharmacopeias occurring naturally and have great scientific and medical value [6]. In the TCM theory system, insect drugs have the effect of activating blood circulation and removing blood stasis, which fits the TCM pathogenesis of HF.

Xinmailong injection (XML, commercialized by the Yunnan Teng Yao Pharmaceutical Co., Ltd, China) is a kind of Chinese patent medicine made by extracting effective substances from Periplaneta americana (a species of cockroach) with modern technology [7]. As a kind of insect drug, fresh adult $P$. americana bodies were dried and processed into powder for the treatment of disease. The P. americana 
powder has a history of applying for thousands of years that can be traced back to the Ming Dynasty (A.D. 1578). It was recorded in the "Compendium of Materia Medica" for its therapeutic effects of promoting blood circulation, detoxification, and urination [8].

Extracts of $P$. americana have many effects, such as promoting wound healing, antitumor activity, and treating $\mathrm{HF}$ and gastrointestinal ulcers. Research studies obtained the chemical compounds from P. americana, which were polyhydric alcohols, organic acids, alkaloids [9], divinyl sulfide, noradrenaline, ketone compounds $[10,11]$, adenosine, inosine, protocatechuic acid, and pyroglutamate acid [12]. These extracts play a specific role in the treatment of diseases with their function of antibacterial, antiviral, and antitumor activity and enhancement of immune function [8]. XML injection as a medicine has a positive inotropic activity, improves microcirculation, dilates pulmonary vessels, induces diuresis, has antiarrhythmia function, and inhibits free radical damage [13].

XML injection has achieved beneficial curative effects in clinical studies and can improve HF patients' cardiac function [14]. However, few studies systematically evaluate the effectiveness of XML injection and the quality of researches. Therefore, we performed this systematic review of available randomized controlled trials to evaluate the efficacy of XML on the improvement of cardiac function in $\mathrm{HF}$ patients. Left ventricular ejection fraction (LVEF), sixminute walk test (6MWT), and brain natriuretic peptide (BNP) were three typical indicators of measuring cardiac functions and were selected as target outcomes in this review. We performed several subgroup analyses and metaregression to find out the facts that caused the heterogeneity among articles.

\section{Materials and Methods}

The present meta-analysis was performed based on the Preferred Reporting Items for Systematic Reviews and MetaAnalyses (PRISMA) guidelines 2015 statement $[15,16]$ and was registered on PROSPERO (CRD42020163716).

2.1. Database and Literature Search Strategies. Online literature search for relevant studies was performed using five databases, which included PubMed, EMBASE, Cochrane Library, Chinese National Knowledge Infrastructure (CNKI), and Wanfang. Studies were reviewed and selected by two experienced investigators. Relevant data were extracted by an additional two independent investigators. Any discrepancies in data extraction or literature review were resolved by consensus or by consulting a third reviewer. All relevant data from the literature review were extracted from the aforementioned databases from inception to April 2020.

The following keywords were searched in various combinations (Xinmailong OR XML OR Xin Mai Long OR XMLI OR Xinmailong Injection) AND (heart failure OR HF OR cardiac failure OR Chronic Heart Failure OR CHF) AND (randomized controlled trial OR RCT).
2.2. Inclusion Criteria. All patients from the selected studies met the internationally accepted criteria for the diagnosis of HF. Only human studies for random clinical trials (RCTs) were considered. No etiology, ethnic group, severity, or course of disease were considered as exclusion criteria. In the experiment group, XML injection plus conventional therapy was considered as a treatment strategy. On the basis of the conventional therapy of western medicine, patients in the control group had placebo or not. Measurable outcomes were LVEF, 6MWT, and BNP.

2.3. Exclusion Criteria. The exclusion criteria were (1) nonRCTs; (2) animal studies, mechanistic studies, case reports, and reviews; (3) patients with acute heart failure and severe liver and kidney disease; (4) use of other TCM formulas; (5) studies with unacceptable trail designs and inappropriate statistical methods; and (6) duplicate publications.

2.4. Data Extraction and Quality Assessment. Clinical data and adverse events were collected and cross-checked by two independent investigators. Any disagreements were resolved through discussion or by consulting a third investigator. The following data were extracted from studies: patient characteristics, details, differences in intervention between the control and experimental groups, outcome measures, and results from the items listed in the inclusion criteria. Methodological quality was assessed based on the Cochrane Handbook. The included RCTs were assessed for (1) random sequence generation, (2) allocation concealment, (3) blinding of participants and personnel, (4) blinding of outcome assessments, (5) selective outcome reporting, (6) incomplete outcome data, and (7) other potential sources of bias [17].

2.5. Data Synthesis. Effect-size calculations and meta-analytical statistics were performed using Stata 12.0 obtained from its official website (http://www.stata.com). Weighted mean differences (WMD) with 95\% CI were expressed for continuous variables and were analyzed using the inversevariance method. Heterogeneity across the trials was evaluated using the Cochran $Q$ test and the Higgins I2 test [18]. Only fixed-effect models were used for both dichotomous and continuous variables, unless the results of pooled analyses showed significant heterogeneity $(P<0.10$ and I2 $>50 \%)$. A random-effect model was used for significant heterogeneity. Potential publication bias was assessed by Begg's and Egger's tests [19]. A 2-tailed $P$ value of less than 0.05 was set for statistical significance. Subgroup analyses and metaregression were performed for sensitivity analysis. Metaregression sensitivity analyses were performed using the "metareg" macro available in the Stata statistical package. Gender, average age, age section, pattern identification, duration time, course of disease, publication year, and sample size were set as the covariances of metaregression analyses. 


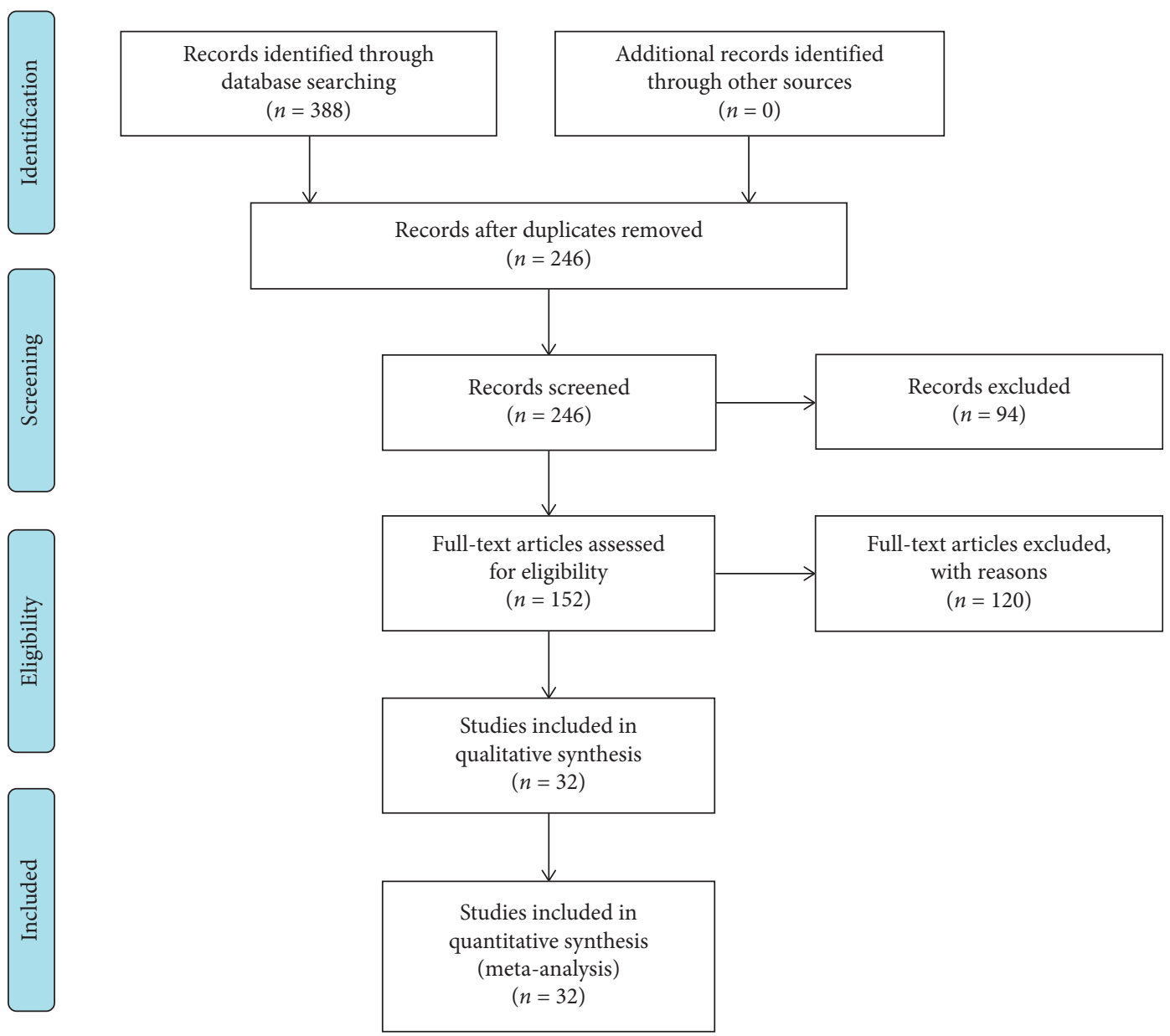

FIGURE 1: Flowchart depicting publication search criteria and selection.

\section{Results}

3.1. Description of Selected Studies. 388 potentially eligible publications were retrieved after the primary search from the eight databases. Of which 32 RCTs were included for the meta-analysis (Figure 1). Except for one selected study that was published in English, the rest studies were published in Chinese. All selected studies were conducted in China and included 3730 patients. Characteristics of the included studies are listed in Table 1.

As shown in Table 2, all 32 selected studies mentioned random sequence generation and no selective reporting bias and 9 studies mentioned allocation sequence and concealment using random number tables. Three studies cited blinding of participants and personnel, and no study mentioned blinding of outcome assessments. 4 studies reported patient attrition; among these, 2 studies reported no dropouts and 2 studies mentioned attrition rates.

\subsection{Results of Outcomes}

3.2.1. Left Ventricular Ejection Fraction (LVEF). Twenty-seven studies reported the LVEF level; the results showed significant increase in LVEF in HF patients with
$\mathrm{XML}$ injection $\quad(\mathrm{WMD}=5.82, \quad 95 \% \quad \mathrm{CI}: \quad 5.52-7.13$, $P<0.00001$ ) (Figure 2). Subgroup analyses showed significantly higher LVEF improvement $(P=0.006)$ in HF patients of middle age (WMD $=7.7,95 \% \mathrm{CI}$ : 5.6-9.79) than those of youth $(\mathrm{WMD}=4.11,95 \% \mathrm{CI}: 2.61-5.62)$ (Table 3 ). However, there was no significant difference $(P=0.88)$ between studies performing TCM pattern identification $(\mathrm{WMD}=5.68,95 \% \mathrm{CI}: 3.83-7.53)$ and those not performing TCM pattern identification $(\mathrm{WMD}=5.87,95 \% \mathrm{CI}$ : 4.38-7.36). There was no publication bias according to Begg's test $(P=0.505)$ and Egger's test $(P=0.109)$ (Table 4$)$.

3.2.2. Six-Minute Walk Test (6MWT). Fifteen studies reported the 6MWT; the results showed significant increase in $6 \mathrm{MWT}$ in HF patients with XML injection (WMD $=51.48$, 95\% CI: 35.83-67.13, $P<0.00001$ ) (Figure 3). Subgroup analysis indicated that XML injection has more 6MWT improvement for $\mathrm{HF}$ patients of middle age $(\mathrm{WMD}=77.39$, 95\% CI: 58.94-95.84) than those of youth (WMD $=34.58$, 95\% CI: $21.26-47.89, P<0.0002$ for between subgroups) (Table 3), but similar increase in pattern identification (WMD $=60.6,95 \% \mathrm{CI}:-1.01$ to 122.21 for pattern identification and $\mathrm{WMD}=48.88,95 \% \mathrm{CI}$ : $34.52-63.24$ for nonpattern identification, $P=0.72$ for between subgroups). No 
TABLE 1: Characteristics of the selected studies.

\begin{tabular}{|c|c|c|c|c|c|c|c|c|c|}
\hline Study & Indication & NYHA & $\begin{array}{l}\text { Sample size } \\
\text { (E/C) }\end{array}$ & $\begin{array}{l}\text { Mean age } \\
\text { (year) }\end{array}$ & $\begin{array}{c}\text { Male/ } \\
\text { female (E/ } \\
\text { C) } \\
\end{array}$ & $\begin{array}{c}\text { Course of } \\
\text { disease (year) }\end{array}$ & Intervention & $\begin{array}{l}\text { Duration } \\
\text { (day) }\end{array}$ & Endpoints \\
\hline Chen 2012 [20] & $\mathrm{CHF}$ & III-IV & $47 / 53$ & $69.3 \pm 6.9$ & NA & $5.7 \pm 1.2$ & $5 \mathrm{mg} / \mathrm{kg}$ bid & 5 & LVEF, BNP \\
\hline $\begin{array}{c}\text { Du et al. } 2016 \\
\text { [21] }\end{array}$ & $\mathrm{CHF}$ & II-IV & $49 / 49$ & $49-79$ & $53 / 45$ & NA & $5 \mathrm{mg} / \mathrm{kg}$ bid & 10 & $\begin{array}{c}\text { LVEF, BNP, } \\
6 \mathrm{MWT}\end{array}$ \\
\hline $\begin{array}{l}\text { Guo and Ren } \\
2016 \text { [22] }\end{array}$ & $\mathrm{CHF}$ & II-IV & $52 / 52$ & $\begin{array}{l}\mathrm{E}: 69 \pm 8 \\
\mathrm{C}: 68 \pm 5\end{array}$ & $\begin{array}{l}\text { E: } 26 / 26 \\
\text { C: } 28 / 24\end{array}$ & $\begin{array}{l}\text { E: } 8.3 \pm 6.0 \\
\text { C: } 8.3 \pm 6.1\end{array}$ & $6 \mathrm{ml}$ bid & 10 & $\begin{array}{l}\text { 6MWT, } \\
\text { LVEF, BNP }\end{array}$ \\
\hline $\begin{array}{l}\text { Han et al. } 2012 \\
\text { [23] }\end{array}$ & $\mathrm{CHF}$ & II-IV & $25 / 21$ & $\begin{array}{l}\mathrm{E}: 65 \pm 7 \\
\mathrm{C}: 68 \pm 6\end{array}$ & $\begin{array}{l}\text { E: } 15 / 10 \\
C: 13 / 8\end{array}$ & NA & $\begin{array}{c}5-10 \mathrm{mg} / \mathrm{kg} \\
\text { bid }\end{array}$ & 14 & $\begin{array}{l}\text { LVEF, } \\
6 \mathrm{MWT}\end{array}$ \\
\hline $\begin{array}{l}\text { Han and Gu } \\
2016[24]\end{array}$ & $\mathrm{CHF}$ & NA & $136 / 147$ & $\begin{array}{l}\text { E: } 79 \pm 11 \\
\text { C: } 77 \pm 12\end{array}$ & $\begin{array}{l}\text { E: } 109 / 27 \\
\text { C: } 122 / 25\end{array}$ & NA & $4 \mathrm{ml}$ bid & 14 & LVEF, BNP \\
\hline $\begin{array}{c}\text { Han and Liu } \\
2018[25]\end{array}$ & $\mathrm{CHF}$ & II-IV & $56 / 56$ & $\begin{array}{l}\text { E: } 72.5 \pm 11.4 \\
\text { C: } 74.3 \pm 12.7\end{array}$ & $\begin{array}{l}\text { E: } 38 / 18 \\
\text { C: } 36 / 20\end{array}$ & NA & $5 \mathrm{mg} / \mathrm{kg}$ bid & 10 & LVEF \\
\hline He 2017 [26] & $\mathrm{CHF}$ & II-IV & $47 / 48$ & $\begin{array}{l}\text { E: } 70.8 \pm 7.6 \\
\text { C: } 69.6 \pm 7.9\end{array}$ & $\begin{array}{l}\text { E: } 23 / 24 \\
\text { C: } 23 / 25\end{array}$ & NA & $5 \mathrm{mg} / \mathrm{kg}$ bid & 15 & $\begin{array}{l}\text { LVEF, } \\
6 \mathrm{MWT}\end{array}$ \\
\hline $\begin{array}{l}\mathrm{Li} \text { and } \mathrm{Li} 2015 \\
{[27]}\end{array}$ & $\mathrm{CHF}$ & II-IV & $35 / 30$ & $\begin{array}{l}\text { E: } 62 \pm 10 \\
\text { C: } 58 \pm 8\end{array}$ & $\begin{array}{l}\text { E: } 20 / 15 \\
\text { C: } 18 / 12\end{array}$ & $\mathrm{NA}$ & $8 \mathrm{ml}$ bid & 15 & LVEF \\
\hline Li 2016 [28] & $\mathrm{CHF}$ & I-IV & $24 / 24$ & $\begin{array}{l}\text { E: } 63.3 \pm 5.8 \\
\text { C: } 63.7 \pm 5.2\end{array}$ & $\begin{array}{l}\text { E: } 16 / 8 \\
\text { C: } 15 / 9\end{array}$ & $\begin{array}{l}\text { E: } 7.6 \pm 6.5 \\
\text { C: } 7.9 \pm 6.3\end{array}$ & $5 \mathrm{mg} / \mathrm{kg}$ bid & 14 & BNP, LVEF \\
\hline $\begin{array}{l}\text { Li et al. } 2018 \\
\text { [29] }\end{array}$ & $\mathrm{HF}$ & II-III & $100 / 100$ & $\begin{array}{l}\text { E: } 58.9 \pm 7.2 \\
\text { C: } 58.4 \pm 7.1\end{array}$ & $\begin{array}{l}\text { E: } 52 / 48 \\
\text { C: } 54 / 46\end{array}$ & $\begin{array}{l}\text { E: } 3.4 \pm 1.4 \\
\text { C: } 3.4 \pm 1.5\end{array}$ & $5 \mathrm{mg} / \mathrm{kg}$ bid & 5 & BNP \\
\hline $\begin{array}{l}\text { Liu HL } 2018 \\
{[30]}\end{array}$ & $\mathrm{CHF}$ & II-IV & $80 / 42$ & $\begin{array}{c}\text { E: } 67 \pm 8 \\
\text { C: } 68 \pm 10\end{array}$ & $\begin{array}{l}\text { E: } 38 / 42 \\
\text { C: } 32 / 48\end{array}$ & NA & $5 \mathrm{mg} / \mathrm{kg}$ bid & 5 & $\begin{array}{l}\text { LVEF, } \\
6 \mathrm{MWT}\end{array}$ \\
\hline Liu et al. 2018 & $\mathrm{CHF}$ & II-IV & $46 / 46$ & $\begin{array}{l}\mathrm{E}: 60.3 \pm 4.0 \\
\mathrm{C}: 61.0 \pm 4.3\end{array}$ & $\begin{array}{l}\text { E: } 25 / 21 \\
\text { C: } 27 / 19\end{array}$ & $\begin{array}{l}\text { E: } 7.9 \pm 1.4 \\
\text { C: } 8.0 \pm 1.3\end{array}$ & $4 \mathrm{ml}$ bid & 14 & BNP, LVEF \\
\hline Liu et al. 2018 & $\mathrm{CHF}$ & II-IV & $60 / 60$ & $\begin{array}{l}\text { E: } 62-84 \\
\text { C: } 63-85\end{array}$ & $\begin{array}{l}\text { E: } 31 / 29 \\
\text { C: } 30 / 30\end{array}$ & NA & $5 \mathrm{mg} / \mathrm{kg}$ bid & 10 & BNP \\
\hline $\begin{array}{l}\text { Quan and Miao } \\
2017[33]\end{array}$ & $\mathrm{CHF}$ & II-IV & $46 / 48$ & $\begin{array}{l}\text { E: } 67.6 \pm 10.5 \\
\text { C: } 65.8 \pm 11.4\end{array}$ & $\begin{array}{l}\text { E: } 35 / 16 \\
\text { C: } 38 / 13\end{array}$ & $\begin{array}{l}\text { E: } 37.8 \pm 7.5 \\
\text { C: } 39.6 \pm 8.6\end{array}$ & $5 \mathrm{mg} / \mathrm{kg}$ bid & 10 & $\begin{array}{l}\text { LVEF, } \\
6 \mathrm{MWT}\end{array}$ \\
\hline $\begin{array}{l}\text { Shen et al. } 2017 \\
\text { [34] }\end{array}$ & $\mathrm{CHF}$ & II-IV & $58 / 58$ & $\begin{array}{l}\text { E: } 62.8 \pm 7.1 \\
\text { C: } 61.6 \pm 7.8\end{array}$ & $\begin{array}{l}\text { E: } 34 / 24 \\
\text { C: } 36 / 22\end{array}$ & $\begin{array}{l}\text { E: } 8.3 \pm 7.5 \\
\text { C: } 8.1 \pm 7.8\end{array}$ & $4 \mathrm{ml}$ bid & 14 & $\begin{array}{l}\text { 6MWT, } \\
\text { LVEF }\end{array}$ \\
\hline Shi et al. 2016 & $\mathrm{HF}$ & NA & $58 / 58$ & $\begin{array}{l}\text { E: } 56.2 \pm 8.74 \\
\text { C: } 55.6 \pm 9.18\end{array}$ & $\begin{array}{l}\text { E: } 28 / 30 \\
\text { C: } 29 / 29\end{array}$ & NA & $5 \mathrm{mg} / \mathrm{kg}$ bid & 5 & BNP \\
\hline $\begin{array}{l}\text { Teng and Wang } \\
2017[36]\end{array}$ & $\mathrm{CHF}$ & II-III & $40 / 40$ & $\begin{array}{l}\text { E: } 75.5 \pm 3.8 \\
\text { C: } 73.4 \pm 3.7\end{array}$ & $\begin{array}{l}\text { E: } 22 / 18 \\
\text { C: } 23 / 17\end{array}$ & $\begin{array}{l}\text { E: } 1.7 \pm 0.5 \\
\text { C: } 1.5 \pm 0.3\end{array}$ & $5 \mathrm{mg} / \mathrm{kg}$ bid & 5 & LVEF \\
\hline $\begin{array}{l}\text { Wang } \\
\text { et al. } \\
2012 \text { [37] }\end{array}$ & III-IV & $24 / 26$ & NA & NA & NA & NA & 10 & $\begin{array}{l}\text { LVEF, } \\
6 \mathrm{MWT} \text {, } \\
\text { BNP }\end{array}$ & \\
\hline $\begin{array}{l}\text { Wu et al. } 2017 \\
{[38]}\end{array}$ & $\mathrm{HF}$ & NA & $48 / 42$ & $\begin{array}{c}\mathrm{E}: \\
54.05 \pm 3.96 \\
\mathrm{C}: \\
56.13 \pm 4.87\end{array}$ & $\begin{array}{l}\text { E: } 28 / 20 \\
\text { C: } 31 / 11\end{array}$ & $\begin{array}{l}\mathrm{E}: 6.33 \pm 0.94 \\
\mathrm{C}: 6.01 \pm 0.33\end{array}$ & $5 \mathrm{mg} / \mathrm{kg}$ bid & 10 & BNP \\
\hline $\begin{array}{l}\text { Wu and Yang } \\
2015 \text { [39] }\end{array}$ & $\mathrm{HF}$ & III-IV & $50 / 50$ & $\begin{array}{l}\text { E: } 70 \pm 7.5 \\
\text { C: } 71 \pm 7.1\end{array}$ & $\begin{array}{l}\text { E: } 30 / 20 \\
\text { C: } 29 / 21\end{array}$ & NA & $5 \mathrm{mg} / \mathrm{kg}$ bid & 10 & BNP \\
\hline $\mathrm{Xu}$ and $\mathrm{Xu} 2016$ & $\mathrm{CHF}$ & NA & $76 / 76$ & NA & $\begin{array}{l}\text { E: } 50 / 26 \\
\text { C: } 48 / 28\end{array}$ & NA & $5 \mathrm{mg} / \mathrm{kg}$ bid & 14 & $\begin{array}{l}\text { LVEF, } \\
6 \mathrm{MWT}\end{array}$ \\
\hline $\begin{array}{l}\text { Xue et al. } 2015 \\
\text { [41] }\end{array}$ & $\mathrm{CHF}$ & II-III & $120 / 115$ & $\begin{array}{l}\mathrm{E}: 63.1 \pm 9.80 \\
\mathrm{C}: 63.9 \pm 9.01\end{array}$ & $\begin{array}{l}\text { E: } 69 / 46 \\
\text { C: } 60 / 60\end{array}$ & $\begin{array}{l}\text { E: } 2.07 \\
\text { C: } 2.37\end{array}$ & $5 \mathrm{mg} / \mathrm{kg}$ bid & 5 & $\begin{array}{l}\text { LVEF, } \\
6 \mathrm{MWT}\end{array}$ \\
\hline $\begin{array}{l}\text { Xue et al. } \\
2019 \text { [14] }\end{array}$ & II-III & $50 / 50$ & $\begin{array}{c}\text { E: } \\
63.8 \pm 9.46 \\
\text { C: } \\
64.3 \pm 7.78\end{array}$ & $\begin{array}{l}\text { E: } 29 / 21 \\
\text { C: } 27 / 23\end{array}$ & $\begin{array}{c}\mathrm{E}: \\
2.48 \pm 2.25 \\
\mathrm{C}: \\
1.90 \pm 1.65\end{array}$ & $5 \mathrm{mg} / \mathrm{kg}$ bid & 16 & $\begin{array}{l}\text { LVEF, } \\
6 \mathrm{MWT}, \\
\text { BNP }\end{array}$ & \\
\hline $\begin{array}{l}\text { Yang } \\
\text { et al. } \\
2012[42]\end{array}$ & III-IV & $57 / 53$ & $\begin{array}{l}\text { E: } 79 \pm 10 \\
\text { C: } 78 \pm 11\end{array}$ & $\begin{array}{c}\text { E: } 46 / 11 \\
\text { C: } 44 / 9\end{array}$ & NA & $4 \mathrm{ml}$ bid & 14 & LVEF, BNP & \\
\hline $\begin{array}{l}\text { Yang et al. } 2017 \\
{[43]}\end{array}$ & $\mathrm{CHF}$ & II-IV & $33 / 33$ & $\begin{array}{l}\text { E: } 60.2 \pm 5.8 \\
\text { C: } 61.1 \pm 4.5\end{array}$ & $\begin{array}{l}\text { E: } 18 / 15 \\
\text { C: } 19 / 14\end{array}$ & $\begin{array}{l}\text { E: } 3.9 \pm 1.2 \\
\text { C: } 3.9 \pm 1.0\end{array}$ & $\begin{array}{c}5-10 \mathrm{mg} / \mathrm{kg} \\
\text { bid }\end{array}$ & 14 & LVEF \\
\hline $\begin{array}{l}\text { Yuan et al. } 2015 \\
\text { [44] }\end{array}$ & $\mathrm{CHF}$ & I-IV & $54 / 34$ & $\begin{array}{l}\text { E: } 51.5 \pm 5.6 \\
\text { C: } 52.3 \pm 6.0\end{array}$ & $\begin{array}{l}\text { E: } 30 / 24 \\
\text { C: } 19 / 15\end{array}$ & $\begin{array}{l}\text { E: } 2.5 \pm 2.3 \\
\text { C: } 2.8 \pm 3.1\end{array}$ & $5 \mathrm{mg} / \mathrm{kg}$ bid & 5 & $6 \mathrm{MWT}$ \\
\hline
\end{tabular}


TABLE 1: Continued.

\begin{tabular}{|c|c|c|c|c|c|c|c|c|c|}
\hline Study & Indication & NYHA & $\begin{array}{l}\text { Sample size } \\
(\mathrm{E} / \mathrm{C})\end{array}$ & $\begin{array}{l}\text { Mean age } \\
\text { (year) }\end{array}$ & $\begin{array}{c}\text { Male/ } \\
\text { female (E/ } \\
\text { C) }\end{array}$ & $\begin{array}{c}\text { Course of } \\
\text { disease (year) }\end{array}$ & Intervention & $\begin{array}{c}\text { Duration } \\
\text { (day) }\end{array}$ & Endpoints \\
\hline $\begin{array}{c}\text { Zhao et al. } 2014 \\
\text { [45] }\end{array}$ & $\mathrm{CHF}$ & II-IV & $30 / 30$ & $\begin{array}{l}\text { E: } 41-78 \\
\text { C: } 40-76\end{array}$ & $\begin{array}{l}\text { E: } 13 / 17 \\
\text { C: } 16 / 14\end{array}$ & NA & $5 \mathrm{mg} / \mathrm{kg}$ bid & 14 & $\begin{array}{l}\text { LVEF, } \\
\text { 6MWT }\end{array}$ \\
\hline $\begin{array}{l}\text { Zhao et al. } 2010 \\
{[46]}\end{array}$ & $\mathrm{CHF}$ & IV & $131 / 112$ & NA & NA & NA & $\begin{array}{c}5-10 \mathrm{mg} / \mathrm{kg} \\
\text { bid }\end{array}$ & 14 & LVEF, BNP \\
\hline $\begin{array}{c}\text { Jiang et al. } 2019 \\
{[47]}\end{array}$ & $\mathrm{HF}$ & l & $52 / 56$ & $\begin{array}{c}\text { E: } 63.1 \pm 7.2 \\
\text { C: } 62.7 \pm 7.6 \\
\text { E: }\end{array}$ & $\begin{array}{l}\text { E: } 29 / 23 \\
\text { C: } 31 / 25\end{array}$ & $\begin{array}{l}\mathrm{E}: 7.87 \pm 4.2 \\
\mathrm{C}: 7.57 \pm 5.7\end{array}$ & $5 \mathrm{mg} / \mathrm{kg}$ bid & 10 & $\begin{array}{l}\text { 6MWT, } \\
\text { LVEF }\end{array}$ \\
\hline Li 2019 [48] & $\mathrm{CHF}$ & II-IV & $200 / 60$ & $\begin{array}{c}63.79 \pm 8.82 \\
\mathrm{C}: \\
62.96 \pm 9.34\end{array}$ & $\begin{array}{l}\text { E: } 131 / 69 \\
\text { C: } 40 / 20\end{array}$ & $\begin{array}{l}\text { E: } 9.06 \pm 2.42 \\
\text { C: } 8.96 \pm 2.25\end{array}$ & $8 \mathrm{ml} \mathrm{qd}$ & 14 & LVEF \\
\hline $\begin{array}{l}\text { Liu and Zhao } \\
2019 \text { [49] }\end{array}$ & $\mathrm{CHF}$ & I-IV & $42 / 42$ & $\begin{array}{c}\text { E: } 57.43 \pm 6 \\
45 \\
\text { C: } 56.55 \pm 5 . \\
94\end{array}$ & $\begin{array}{l}\text { E: } 25 / 17 \\
\text { C: } 20 / 22\end{array}$ & $\mathrm{NA}$ & $\begin{array}{c}5-10 \mathrm{mg} / \mathrm{kg} \\
\text { bid }\end{array}$ & 5 & LVEF \\
\hline $\begin{array}{c}\text { Zhang and } \\
\text { Zhao } 2019 \text { [50] }\end{array}$ & $\mathrm{CHF}$ & II-IV & $47 / 46$ & $\begin{array}{c}\mathrm{E}: \\
68.85 \pm 6.20 \\
\mathrm{C}: \\
69.03 \pm 6.31\end{array}$ & $\begin{array}{l}\text { E: } 27 / 20 \\
\text { C: } 28 / 18\end{array}$ & NA & $5 \mathrm{mg} / \mathrm{kg}$ bid & 14 & LVEF \\
\hline
\end{tabular}

NYHA, New York Heart Association; E, experimental group; C, control group; CHF, chronic heart failure; HF, heart failure, NA, not applicable; BNP, brain natriuretic peptide; 6MWT, 6-minute walk test; LVEF, left ventricular ejection fraction; IVST, interventricular septal thickness.

TABLE 2: Risk of bias.

\begin{tabular}{|c|c|c|c|c|c|c|c|}
\hline Study & $\begin{array}{l}\text { Random } \\
\text { sequence } \\
\text { generation }\end{array}$ & $\begin{array}{l}\text { Allocation } \\
\text { concealment }\end{array}$ & $\begin{array}{c}\text { Blinding of } \\
\text { participants and } \\
\text { personnel }\end{array}$ & $\begin{array}{l}\text { Blinding of } \\
\text { outcome } \\
\text { assessment }\end{array}$ & $\begin{array}{l}\text { Incomplete } \\
\text { outcome data }\end{array}$ & $\begin{array}{l}\text { Selective } \\
\text { reporting }\end{array}$ & $\begin{array}{c}\text { Other } \\
\text { bias }\end{array}$ \\
\hline $\begin{array}{l}\text { Chen } 2012 \\
{[20]}\end{array}$ & + & l & I & l & l & + & I \\
\hline $\begin{array}{l}\text { Du et al. } 2016 \\
\text { [21] }\end{array}$ & + & I & l & 1 & l & + & I \\
\hline $\begin{array}{l}\text { Guo and Ren } \\
2016 \text { [22] }\end{array}$ & + & + & 1 & 1 & I & + & I \\
\hline $\begin{array}{l}\text { Han et al. } \\
2012 \text { [23] }\end{array}$ & + & 1 & 1 & 1 & I & + & 1 \\
\hline $\begin{array}{l}\text { Han and Gu } \\
2016[24]\end{array}$ & + & l & 1 & 1 & l & + & 1 \\
\hline $\begin{array}{l}\text { Han and Liu } \\
2018 \text { [25] }\end{array}$ & + & 1 & I & 1 & I & + & 1 \\
\hline He 2017 [26] & + & I & l & l & l & + & l \\
\hline $\begin{array}{l}\mathrm{Li} \text { and Li } 2015 \\
\text { [27] }\end{array}$ & + & I & I & 1 & l & + & 1 \\
\hline Li 2016 [28] & + & + & l & l & l & + & l \\
\hline $\begin{array}{l}\text { Li et al. } 2018 \\
\text { [29] }\end{array}$ & + & + & I & 1 & I & + & I \\
\hline $\begin{array}{l}\text { Liu et al. } 2018 \\
\text { [30] }\end{array}$ & + & 1 & 1 & I & I & + & 1 \\
\hline Liu 2018 [31] & + & l & I & I & I & + & I \\
\hline $\begin{array}{l}\text { Liu et al. } \\
2018 \text { [32] }\end{array}$ & + & I & I & I & I & + & I \\
\hline Quan and & & & & & & & \\
\hline $\begin{array}{l}\text { Miao } 2017 \\
{[33]}\end{array}$ & + & + & I & I & + & + & l \\
\hline $\begin{array}{l}\text { Shen et al. } \\
2017 \text { [34] }\end{array}$ & + & I & + & l & I & + & I \\
\hline $\begin{array}{l}\text { Shi et al. } 2016 \\
\text { [35] }\end{array}$ & + & l & I & l & I & + & I \\
\hline
\end{tabular}


TABLE 2: Continued.

\begin{tabular}{|c|c|c|c|c|c|c|c|}
\hline Study & $\begin{array}{c}\text { Random } \\
\text { sequence } \\
\text { generation } \\
\end{array}$ & $\begin{array}{c}\text { Allocation } \\
\text { concealment }\end{array}$ & $\begin{array}{c}\text { Blinding of } \\
\text { participants and } \\
\text { personnel }\end{array}$ & $\begin{array}{c}\text { Blinding of } \\
\text { outcome } \\
\text { assessment }\end{array}$ & $\begin{array}{l}\text { Incomplete } \\
\text { outcome data }\end{array}$ & $\begin{array}{l}\text { Selective } \\
\text { reporting }\end{array}$ & $\begin{array}{c}\text { Other } \\
\text { bias }\end{array}$ \\
\hline \multicolumn{8}{|l|}{ Teng and } \\
\hline $\begin{array}{l}\text { Wang } 2017 \\
{[36]}\end{array}$ & + & + & I & I & l & + & I \\
\hline $\begin{array}{l}\text { Wang et al. } \\
2012 \text { [37] }\end{array}$ & + & I & I & I & - & + & l \\
\hline $\begin{array}{l}\text { Wu et al. } 2017 \\
\text { [38] }\end{array}$ & + & + & I & I & I & + & I \\
\hline $\begin{array}{l}\text { Wu and Yang } \\
2015 \text { [39] }\end{array}$ & + & I & I & I & l & + & l \\
\hline $\begin{array}{l}\mathrm{Xu} \text { and } \mathrm{Xu} \\
2016[40]\end{array}$ & + & I & I & I & I & + & I \\
\hline $\begin{array}{l}\text { Xue et al. } \\
2015 \text { [41] }\end{array}$ & + & + & + & I & - & + & l \\
\hline $\begin{array}{l}\text { Xue et al. } \\
2019 \text { [14] }\end{array}$ & + & + & + & I & + & + & I \\
\hline $\begin{array}{l}\text { Yang et al. } \\
2012 \text { [42] }\end{array}$ & + & I & 1 & 1 & I & + & l \\
\hline $\begin{array}{l}\text { Yang et al. } \\
2017 \text { [43] }\end{array}$ & + & I & I & I & I & + & I \\
\hline $\begin{array}{l}\text { Yuan et al. } \\
2015 \text { [44] }\end{array}$ & + & l & I & I & l & + & I \\
\hline $\begin{array}{l}\text { Zhao et al. } \\
2014 \text { [45] }\end{array}$ & + & I & I & I & l & + & I \\
\hline $\begin{array}{l}\text { Zhao et al. } \\
2010 \text { [46] }\end{array}$ & + & I & 1 & I & l & + & I \\
\hline $\begin{array}{l}\text { Jiang et al. } \\
2019 \text { [47] }\end{array}$ & + & I & I & I & I & + & I \\
\hline Li 2019 [48] & + & I & l & I & l & + & I \\
\hline $\begin{array}{l}\text { Liu and Zhao } \\
2019 \text { [49] }\end{array}$ & + & I & I & l & I & + & I \\
\hline Zhang and & & & & & & & \\
\hline $\begin{array}{l}\text { Zhao } 2019 \\
{[50]}\end{array}$ & + & + & 1 & l & l & + & l \\
\hline
\end{tabular}

+, low risk; -, high risk; /, unclear risk.

publication bias was observed in the 6MWT of HF patients ( $P=0.451$ for Begg's test and $P=0.209$ for Egger's test) (Table 4).

3.2.3. Brain Natriuretic Peptide (BNP). Sixteen studies reported the BNP; the results showed that BNP level in the XML plus conventional therapy group was significantly higher compared to conventional therapy alone (WMD $=-172.84, \quad 95 \% \quad \mathrm{CI}:-205.79$ to -139.89 , $P=0.00001$ ) (Figure 4). For the subgroup analysis based on age section, significantly higher BNP decrease was observed in middle-aged $\mathrm{HF}$ patients than young patients (WMD $=-301.54,95 \% \mathrm{CI}:-405.38$ to -197.71 for middleaged and $\mathrm{WMD}=-156.7,95 \% \mathrm{CI}:-201.26$ to -112.15 for youth, $P=0.01$ for between subgroups) (Table 3 ). No significant difference was observed in subgroup analysis based on pattern identification $(\mathrm{WMD}=-243.87,95 \% \mathrm{CI}$ : -426.98 to -60.76 for pattern identification and $\mathrm{WMD}=-159.1,95 \% \mathrm{CI}:-193.3$ to -124.89 for nonpattern identification, $P=0.37$ for between subgroups). The results of Begg's test $(P=0.137)$ and Egger's test $(P=0.014)$ indicated potential publication bias in the BNP level (Table 4).

3.2.4. Metaregression Results. The results of metaregression (Table 5) suggested that the age section and average age could be two main sources that contributed to the heterogeneity of LVEF and 6MWT. Unfortunately, gender, pattern identification, duration time, course of the disease, publication year, and sample size showed no significant effect on LVEF and 6MWT, and none of these indicators listed had a statistically significant effect on the BNP level. The significant regression effect of age section (adj $R^{2}=26.75 \%, \quad P=0.013$ for LVEF; adj $R^{2}=69.25 \%$, $P=0.003$ for $6 \mathrm{MWT}$ ) met the results of subgroup analyses, which further indicated that middle-aged HF patients with XML administration could have higher LVEF and 6MWT improvement than the youth. The metaregression results based on average age (adj $R^{2}=16.54 \%, P=0.049$ for LVEF; adj $R^{2}=36.39 \%, P=0.048$ for $6 \mathrm{MWT}$ ) suggested that greater LVEF and 6MWT improvements were associated with higher average age (Figure 5). 


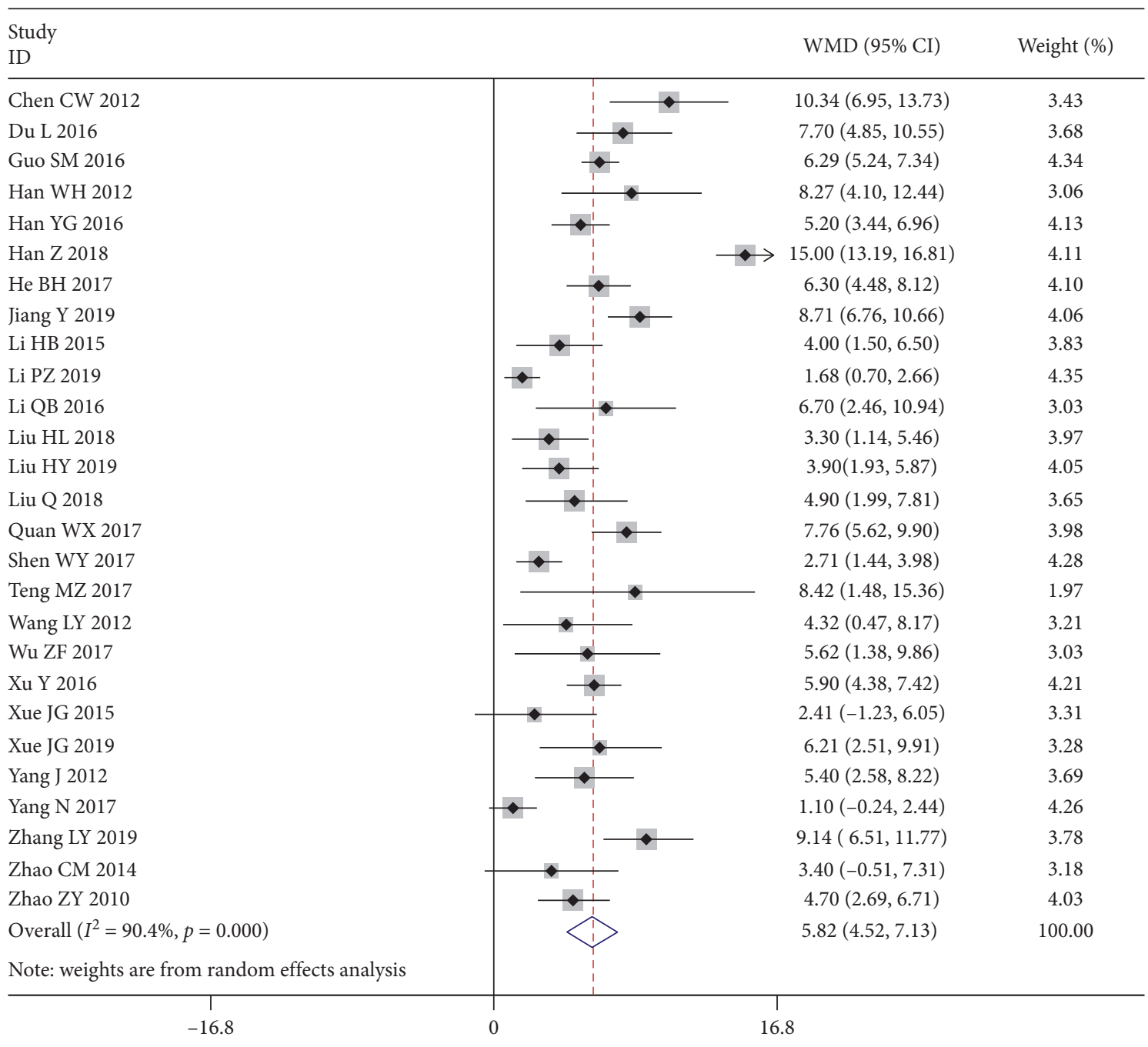

FIGURE 2: Forest plot of LVEF in patients administered XML plus conventional therapy versus conventional HF therapy alone.

3.2.5. Adverse Events. 16 out of the 32 studies investigated adverse events. 10 of these studies reported no obvious adverse events, while the remaining 6 studies mentioned details of adverse events. As shown in Table 6, 7 adverse events were observed between the experimental and control groups. These included cutaneous pruritus (3/0), palpitation $(1 / 4)$, dizziness $(2 / 0)$, headaches $(0 / 1)$, nausea $(2 / 1)$, leukocytosis $(0 / 2)$, and hypotension (1/0). All symptoms reported were resolved during study observation, and no deaths related to XML administration were reported.

\section{Discussion}

4.1. Summary of Main Results. XML injection is a bioactive composite extracted from $P$. americana. The injection form can avoid people's visual aversion and improve the utilization ratio of bioavailability for its advanced extraction process. XML injection has been widely used in China for decades. Studies provided evidences to support XML as an effective administration to HF patients that XML injection can mitigate epirubicin-induced cardiotoxicity via activating the PI3K/Akt signaling pathway and inhibiting the Erk1/2 and P38 MAPK signaling pathways [51]. XML can also activate $T$-type calcium channels and inhibit $\mathrm{Na}+/ \mathrm{K}+-$ ATPase to increase intracellular calcium levels when treating HF [52]. Besides, the pharmacological research on XML has being carried out continuously and many components and functions of XML are being explored [12]. Despite its treatment effects on HF, it can also repair damaged skin and treat gastric ulcers [8].

This systematic review provided evidence that additional XML injection treatment demonstrates better therapeutic effects than conventional treatment on improving cardiac function in patients with $\mathrm{HF}$ evidenced by the increase in LVEF and 6MWT and reduction in BNP. Subgroup analyses provided evidence that the age section is a source of causing significant differences between groups. Under XML injection administration, the cardiac function of middle-aged patients showed better improvement than young patients (average $>65$ as middle aged, average $<65$ as youth). Further metaregression demonstrated that age section and average age showed significant heterogeneity in LVEF and 6MWT and 
TABLE 3: Mean difference of cardiac function of HF patients with XML injection administration in different subgroup analyses.

\begin{tabular}{|c|c|c|c|c|c|c|c|c|c|c|c|c|}
\hline & \multicolumn{4}{|c|}{ LVEF } & \multicolumn{4}{|c|}{ 6MWT } & \multicolumn{4}{|c|}{$\mathrm{BNP}$} \\
\hline & $N$ & $\begin{array}{c}\text { WMD }(95 \% \\
\text { CI })\end{array}$ & $\begin{array}{l}I^{2} \\
(\%)\end{array}$ & $\begin{array}{l}P \text { value for } \\
\text { between-group } \\
\text { difference }\end{array}$ & $N$ & $\begin{array}{c}\text { WMD } \\
(95 \% \mathrm{CI})\end{array}$ & $\begin{array}{l}I^{2} \\
(\%)\end{array}$ & $\begin{array}{c}P \text { value for } \\
\text { between-group } \\
\text { difference }\end{array}$ & $N$ & $\begin{array}{c}\text { WMD } \\
(95 \% \mathrm{CI})\end{array}$ & $\begin{array}{l}I^{2} \\
(\%)\end{array}$ & $\begin{array}{c}P \text { value for } \\
\text { between-group } \\
\text { difference }\end{array}$ \\
\hline Overall & 27 & $\begin{array}{c}5.82 \\
(4.52,7.13)\end{array}$ & 90 & & 15 & $\begin{array}{c}51.48 \\
(35.83 \\
67.13)\end{array}$ & 88 & & 16 & $\begin{array}{l}-172.84 \\
(-205.79 \\
-139.89)\end{array}$ & 94 & \\
\hline Subgroups & & & & & & & & & & & & \\
\hline Age section & & & & 0.006 & & & & 0.0002 & & & & 0.01 \\
\hline Youth & 11 & $\begin{array}{l}4.11(2.61 \\
5.62)\end{array}$ & 83 & & 6 & $\begin{array}{l}34.58 \\
(21.26 \\
47.89)\end{array}$ & 61 & & 5 & $\begin{array}{l}-156.70 \\
(-201.26 \\
-112.15)\end{array}$ & 88 & \\
\hline Middle age & 11 & $\begin{array}{c}7.70(5.60 \\
9.79)\end{array}$ & 90 & & 5 & $\begin{array}{l}77.39 \\
(58.94 \\
95.84)\end{array}$ & 77 & & 6 & $\begin{array}{l}-301.54 \\
(-405.38 \\
-197.71)\end{array}$ & 97 & \\
\hline $\begin{array}{l}\text { Pattern } \\
\text { identification }\end{array}$ & & & & 0.88 & & & & 0.72 & & & & 0.37 \\
\hline Yes & 4 & $\begin{array}{c}5.68(3.83 \\
7.53)\end{array}$ & 33 & & 3 & $\begin{array}{r}60.60 \\
(-1.01 \\
122.21)\end{array}$ & 87 & & 3 & $\begin{array}{c}-243.87 \\
(-426.98 \\
-60.76)\end{array}$ & 97 & \\
\hline No & 23 & $\begin{array}{c}5.87(4.38 \\
7.36)\end{array}$ & 90 & & 12 & $\begin{array}{l}48.88 \\
(34.52, \\
63.24)\end{array}$ & 84 & & 13 & $\begin{array}{l}-159.10 \\
(-193.30 \\
-124.89)\end{array}$ & 94 & \\
\hline
\end{tabular}

LVEF, left ventricular ejection fraction; 6MWT, six-minute walk test; BNP, brain natriuretic peptide; WMD, weighted mean difference; CI, confidence interval.

TABle 4: Publication bias.

\begin{tabular}{lccc}
\hline & Number of studies & Begg's $P$ value & Egger's $P$ value \\
\hline LVEF & 27 & 0.505 & 0.109 \\
6MWT & 12 & 0.451 & 0.209 \\
BNP & 16 & 0.137 & 0.014 \\
\hline
\end{tabular}

LVEF, left ventricular ejection fraction; 6MWT, six-minute walk test; BNP, brain natriuretic peptide.

greater improvement of LVEF and 6MWT was connected to a higher age. The above evidences lead to the conclusion that XML injection may be more suitable for middle-aged patients and its efficiency increases by age, while it has a considerable improvement in HF patients of all age ranges.

The pattern identification presented no potential heterogeneity in both subgroup analysis and metaregression, indicating XML injection, as a single-substance extract, has fixed therapeutic efficacy on HF without pattern identification, unlike other Chinese patent drugs that focus on TCM syndromes. Unfortunately, the rest covariances (gender, duration time, course of disease, publication year, and sample size) may contribute no significance to the heterogeneity.

4.2. Adverse Events. No obvious adverse reactions of Xinmailong injection were found before marketing. With the expansion of users after marketing, many adverse reactions have been reported gradually. In previous reviews, the incidence of adverse reactions was $7.6 \%$ [53]. The most common symptoms are pruritus, slight blood pressure rise, nausea, and vomiting. In this review, adverse effects of XML injection were also collected from included studies, of which
2 studies mentioned skin itching [14, 36], each study mentioned hypertension [37] and nausea [36]. Other studies also described palpitation, dizziness, headache, and leukocytosis of HF patients during XML administration. There is no case of liver and kidney function damage in the clinical study of Xinmailong. On the contrary, there is a protective effect of XML injection on the early renal function damage caused by grass carp bile [54] and extracts of $P$. americana have a protective effect on liver damage, liver fibrosis, and hepatitis [55]. Although all the adverse reactions mentioned above can be relieved by rest or slowing down the intravenous drip, no lethal events or major adverse cardiovascular events were observed. XML seemed to be generally safe for HF treatment, but the evidence is still insufficient to make a decisive conclusion on safety. Attentions are still needed in practical application, as the safety of TCM herbal injections has been increasingly concerned by both medical workers and the public.

4.3. Limitations. Study limitations in this review were mostly due to the risk of bias [56] of included studies. Many studies have no description of the necessary process of clinical trials, and therefore the evaluation of these studies 


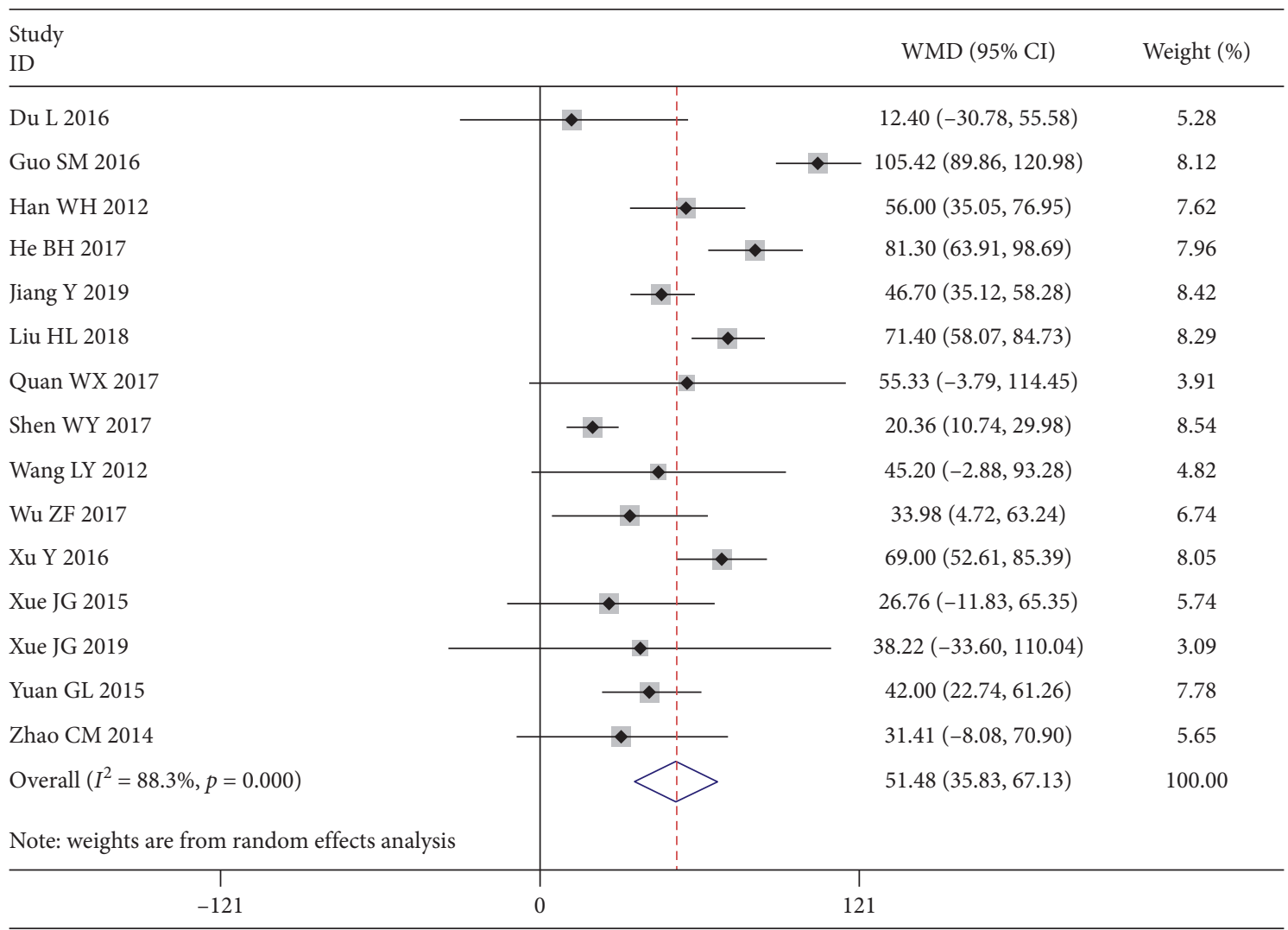

FIGURE 3: Forest plot of 6MWT in patients administered XML plus conventional therapy versus conventional HF therapy alone.

\begin{tabular}{|c|c|c|c|}
\hline \multicolumn{2}{|l|}{$\begin{array}{l}\text { Study } \\
\text { ID }\end{array}$} & WMD $(95 \% \mathrm{CI})$ & Weight (\%) \\
\hline Li ZG 2018 & $\bullet$ & $-145.30(-163.42,-127.18)$ & 8.00 \\
\hline Xue JG 2019 & $\bullet$ & $-72.60(-132.27,-12.93)$ & 6.46 \\
\hline Li QB 2016 & $\bullet$ & $-254.80(-295.26,-214.34)$ & 7.29 \\
\hline Liu Q 2018 & $\bullet$ & $-138.90(-186.47,-91.33)$ & 7.00 \\
\hline Wu ZF 2017 & $\bullet$ & $-158.78(-189.51,-128.05)$ & 7.65 \\
\hline Guo SM 2016 & $\bullet$ & $-530.56(-622.94,-438.18)$ & 4.98 \\
\hline Chen CW 2012 & 1 & $-3270.00(-4826.50,-1713.50)$ & 0.04 \\
\hline Han YG 2016 & $\bullet$ & $-97.20(-112.45,-81.95)$ & 8.05 \\
\hline Liu HL 2018 & $\bullet$ & $-113.10(-154.61,-71.59)$ & 7.25 \\
\hline Wu TK 2015 & 11 & $-1044.00(-1277.39,-810.61)$ & 1.60 \\
\hline Yang J 2012 & $\bullet$ & $-95.40(-120.32,-70.48)$ & 7.83 \\
\hline Du L 2016 & $\bullet$ & $-147.70(-186.85,-108.55)$ & 7.35 \\
\hline Liu YH 2018 & $\bullet$ & $-163.12(-179.16,-147.08$ & 8.04 \\
\hline Shi HR 2016 & $\bullet$ & $-156.80(-183.72,-129.88)$ & 7.77 \\
\hline Wang LY 2012 & 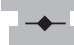 & $-177.71(-344.60,-10.82)$ & 2.64 \\
\hline Zhao ZY 2010 & $\bullet$ & $-88.30(-104.20,-72.40)$ & 8.04 \\
\hline Overall $\left(I^{2}=94.4 \%, p=0.000\right)$ & 8 & $-172.84(-205.79,-139.89)$ & 100.00 \\
\hline \multicolumn{4}{|c|}{ Note: weights are from random effects analysis } \\
\hline $\begin{array}{c} \\
-4826\end{array}$ & 0 & $\begin{array}{c}1 \\
4826\end{array}$ & \\
\hline
\end{tabular}

FIGURE 4: Forest plot of BNP level in patients administered XML plus conventional therapy versus conventional HF therapy alone. 
TABLE 5: Metaregression analysis of association between covariates and cardiac function of HF patients with XML injection administration.

\begin{tabular}{|c|c|c|c|c|c|c|c|c|c|c|c|c|c|c|c|}
\hline & \multicolumn{5}{|c|}{ LVEF } & \multicolumn{5}{|c|}{$6 \mathrm{MWT}$} & \multicolumn{5}{|c|}{$\mathrm{BNP}$} \\
\hline & $N$ & $\begin{array}{c}P \\
\text { value }\end{array}$ & $\mathrm{Tau}^{2}$ & $I^{2}(\%)$ & $\begin{array}{l}\text { Adj } R^{2} \\
(\%)\end{array}$ & $N$ & $\begin{array}{c}P \\
\text { value }\end{array}$ & $\mathrm{Tau}^{2}$ & $I^{2}(\%)$ & $\begin{array}{c}\text { Adj } R^{2} \\
(\%)\end{array}$ & $N$ & $\begin{array}{c}P \\
\text { value }\end{array}$ & $\mathrm{Tau}^{2}$ & $I^{2}(\%)$ & $\begin{array}{c}\text { Adj } R^{2} \\
(\%)\end{array}$ \\
\hline Gender & 22 & 0.727 & 9.304 & 92.03 & -4.89 & 12 & 0.447 & 656.7 & 88.15 & -0.47 & 12 & 0.573 & 61293 & 93.91 & -8.69 \\
\hline Age section & 2 & 0.013 & 7.121 & 87.36 & 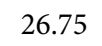 & 11 & 0.003 & 192.5 & 70.52 & 69.25 & 11 & 0.340 & 98464 & 5.34 & -3 \\
\hline Average age & 22 & 0.049 & 8.114 & 89.25 & 6.54 & 11 & 0.048 & 398.2 & 86.05 & 36.39 & 11 & 0.737 & 117450 & 95.15 & -22.93 \\
\hline $\begin{array}{l}\text { Pattern } \\
\text { identification }\end{array}$ & 27 & 0.890 & 8.442 & 90.55 & .97 & 15 & 0.300 & 508 & 84.71 & 8.82 & 16 & 0.924 & 53996 & 94.60 & -12.45 \\
\hline Duration time & 27 & 0.436 & 8.202 & 89.67 & -1.02 & 16 & 0.836 & 558.2 & 87.91 & -8.62 & 16 & 0.549 & 54559 & 93.98 & -13.62 \\
\hline Course of disease & 13 & 0.421 & 7.450 & 89.28 & -1.03 & 8 & 0.686 & 812 & 92.87 & -7.69 & 7 & 0.456 & 20608 & 93.53 & 27.79 \\
\hline Publication year & 27 & 0.884 & 8.464 & 90.61 & -4.24 & 15 & 0.805 & 603.6 & 89.07 & -8.36 & 16 & 0.683 & 55437 & 93.69 & -15.45 \\
\hline Sample size & 27 & 0.255 & 8.014 & 89.78 & 1.31 & 15 & 0.865 & 600.2 & 89.11 & -7.75 & 16 & 0.484 & 49753 & 92.83 & -3.62 \\
\hline
\end{tabular}

LVEF, left ventricular ejection fraction; 6MWT, six-minute walk test; BNP, brain natriuretic peptide.

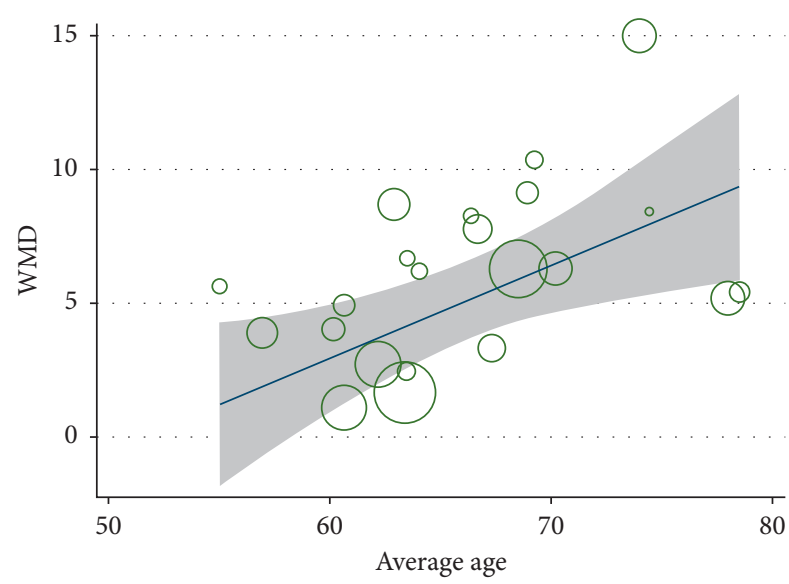

(a)

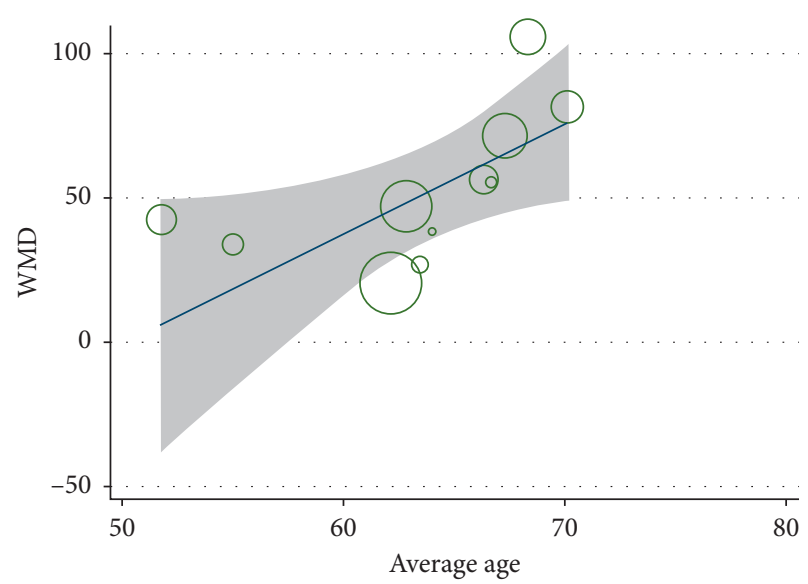

(b)

FIGURE 5: Metaregression bubble plot of correlation between WMD of (a) LVEF and (b) 6MWT and average age.

TABLE 6: Incidence rates for adverse events.

\begin{tabular}{|c|c|c|c|c|}
\hline \multirow{2}{*}{ Items } & \multirow{2}{*}{ No. of studies } & \multicolumn{2}{|c|}{ No. of adverse events } & \multirow{2}{*}{ References } \\
\hline & & $\mathrm{XML}$ & Control & \\
\hline Cutaneous pruritus & 2 & 3 & 0 & Xue et al. 2019 [14], Teng and Wang 2017 [36] \\
\hline Palpitation & 2 & 1 & 4 & Han and Liu 2018 [25], Du et al. 2016 [21] \\
\hline Dizziness & 2 & 2 & 0 & Du et al. 2016 [21], Zhao et al. 2010 [46] \\
\hline Headache & 1 & 0 & 1 & Zhao et al. 2010 [46] \\
\hline Nausea & 1 & 2 & 1 & Teng and Wang 2017 [36] \\
\hline Leukocytosis & 1 & 0 & 2 & Xue et al. 2019 [14] \\
\hline Hypotension & 1 & 1 & 0 & Wang et al. 2012 [37] \\
\hline
\end{tabular}

related to unclear risk of bias. Besides, current research studies focus on the total effect of XML injection, while paying less attention to comprehensive assessment based on basic data collection of different types of patients, which brought difficulty to follow up subgroup analyses and metaregression analysis. Studies in the future could be designed with an emphasis on confirmation and further exploration of age grouping, as average age had been explored to be a source of heterogeneity.

Concerning data extraction, the included trials used different diagnostic and measurement criteria for $\mathrm{HF}$ patients. These different measurement criteria and the use of different instruments affect study comparisons and hence the meta-analysis. Treatment based on pattern identification is a key characteristic of TCM [57]. Application of Chinese patent medicines without the implementation of TCM pattern identification could be a matter of heterogeneity and subsequently reduce the reliability of meta-analysis results. Subgroup analyses in this review, unfortunately, found no heterogeneity based on pattern identification. Further studies are needed for conducting patient selection in which pattern identification is classified.

The results of Begg's and Egger's tests demonstrated no significant asymmetry and suggested no potential 
publication bias in this review [58]. Future clinical studies investigating XML treatment for HF should be designed with appropriate controls and better study designs using large patient cohorts, multicenter and prospective. Heterogeneity within the study should be addressed and include appropriate subgroup classification.

\section{Conclusion}

This review demonstrated that combinational use of XML and conventional treatment may demonstrate better therapeutic effects on improving cardiac function in patients with HF, and age is a potential factor of higher efficacy. Study limitations included the low quality of the selected trials. Additional large, multicenter, prospective clinical trials are warranted to validate our findings.

\section{Data Availability}

The data used to support this systematic review are included within the article.

\section{Conflicts of Interest}

The authors declare that there are no conflicts of interest regarding the publication of this paper.

\section{Authors' Contributions}

Wang XL, Ruan XF, and Sun YL proposed the meta-analysis and designed the study. Qiang TT, Li YP, and Ruan XF performed publication review. Li YP, Sun YL, and Wang XL performed data extraction and analysis. Sun YL wrote the manuscript.

\section{Acknowledgments}

The authors would like to thank the authors of the selected XML clinical trials for performing their work investigating HF treatment strategies. This work was financially supported by the National Natural Science Foundation of China (Nos. 81573647 and 81403352) and the Three-Year Foundation Project of Further Development of Traditional Chinese Medicine in Shanghai (ZY(2018-2020)-CCCX-2003-07).

\section{References}

[1] A. P. Ambrosy, G. C. Fonarow, J. Butler et al., "The global health and economic burden of hospitalizations for heart failure," Journal of the American College of Cardiology, vol. 63, no. 12, pp. 1123-1133, 2014.

[2] E. J. Benjamin, S. S. Virani, C. W. Callaway et al., "Heart disease and stroke statistics-2018 update: a report from the American heart association," Circulation, vol. 137, no. 12, p. E493, 2018.

[3] P. A. Heidenreich, N. M. Albert, L. A. Allen et al., "Forecasting the impact of heart failure in the United States," Circulation: Heart Failure, vol. 6, no. 3, pp. 606-619, 2013.

[4] J. Huang, "Epidemiological characteristics and prevention strategies of heart failure in China," Chinese Journal of Heart and Heart Rhythm, vol. 3, no. 2, pp. 81-82, 2015.
[5] G. Maheedhar, K. Muhammad, and J. Orvar, "Heart failure," South Dakota Medicine the Journal of the South Dakota State Medical Association, vol. 68, no. 9, pp. 403-409, 2015.

[6] U. Bushra, B. Rabia, K. B. Ali, Z. Sarwat, and F. Fehmida, "Potential uses of venom proteins in treatment of HIV," Protein and Peptide Letters, vol. 25, no. 7, pp. 619-625, 2018.

[7] S. Bhattacharjya, I.-W. Kim, J. H. Lee et al., "De novo transcriptome analysis and detection of antimicrobial peptides of the American cockroach Periplaneta americana (linnaeus)," PLoS ONE, vol. 11, p. 5, 2016.

[8] C. Zeng, Q. Liao, Y. Hu, Y. Shen, F. Geng, and L. Chen, "The role of Periplaneta americana (blattodea: blattidae) in modern versus traditional Chinese medicine," Journal of Medical Entomology, vol. 56, no. 6, pp. 1522-1526, 2019.

[9] H. Wang, Y. Ye, W. Wan et al., "Xinmailong modulates platelet function and inhibits thrombus formation via the platelet alphaIIbbeta3-mediated signaling pathway," Frontiers in Pharmacology, vol. 10, p. 923, 2019.

[10] C. X. Jiao, C. G. Zhang, and G. M. Liu, "Gas chromatography-mass spectrometry of the volatile components in the Periplaneta americana alcohol extraction of water-soluble ingredients," Lishizhen Medicine and Materia Medica Research, vol. 23, no. 1, pp. 2797-2798, 2012.

[11] C. X. Jiao, C. G. Zhang, G. M. Liu, and S. N. Li, "Establishment of HPLC fingerprint of Xinmailong injection," Traditional Chinese Patent Medicines, vol. 33, no. 1, pp. 1648-1652, 2011.

[12] J. Qi, J. Yu, Y. Tan et al., "Mechanisms of Chinese Medicine Xinmailong's protection against heart failure in pressureoverloaded mice and cultured cardiomyocytes," Scientific Reports, vol. 7, no. 1, Article ID 42843, 2017.

[13] L. Zhang, "Pharmacological effect and clinical effect of Xinmailong injection," Herald of Medicine, vol. 20, no. 4, p. 250, 2001.

[14] J. Xue, Y. Xu, Y. Deng et al., "The efficacy and safety of Xinmailong injection in patients with chronic heart failure: a multicenter randomized double-blind placebo-controlled trial," The Journal of Alternative and Complementary Medicine, vol. 25, no. 8, pp. 856-860, 2019.

[15] L. Shamseer, D. Moher, M. Clarke et al., "Preferred reporting items for systematic review and meta-analysis protocols (PRISMA-P) 2015: elaboration and explanation," Bmj, vol. 349, no. jan02 1, p. g7647, 2015.

[16] S. Upadhaya, S. Madala, R. Baniya, K. Saginala, and J. Khan, "Impact of acetylsalicylic acid on primary prevention of cardiovascular diseases: a meta-analysis of randomized trials," European Journal of Preventive Cardiology, vol. 26, no. 7, pp. 746-749, 2019.

[17] F. Fang, Y. Zhang, J. Tang et al., "Association of corticosteroid treatment with outcomes in adult patients with sepsis," JAMA Internal Medicine, vol. 179, no. 2, pp. 213-223, 2019.

[18] J. Higgins, "Measuring inconsistency in meta-analyses," Bmj, vol. 327, no. 7414, pp. 557-560, 2003.

[19] G. D. S. Egger, M. Schneider, and C. E. Minder, "Bias in metaanalysis detected by a simple, graphical test,” Bmj, vol. 315, no. 7109, pp. 629-634, 1997.

[20] C. W. Chen, "Clinical observation of Xinmailong injection in the treatment of chronic congestive heart failure," Journal of Qiqihar Medical University, vol. 33, no. 18, pp. 2461-2462, 2012.

[21] L. Du, Y. Wu, W. Li, F. Hu, G. Y. Ge, and X. N. Sun, “Clinical effect of Xinmailong injection on chronic heart failure and its effect on cardiac function and BNP," Chinese Journal of Integrative Medicine on Cardio-Cerebrovascular Disease, vol. 14, no. 22, pp. 2656-2658, 2016. 
[22] S. M. Guo and J. M. Ren, "Effects of different doses of Xinmailong injection on cardiac function and plasma B-type natriuretic peptide in elderly patients with chronic heart failure," Herald of Medicine, vol. 35, no. 01, pp. 54-57, 2016.

[23] W. H. Han, G. Li, S. . i. Wang, X. X. He, and S. J. Yang, "Clinical observation of Xinmailong injection in the treatment of senile chronic heart failure," Chinese Journal of Cardiovascular Rehabilitation Medicine, vol. 21, no. 04, pp. 422-424, 2012.

[24] Y. Han and H. Gu, "Effect and significance of conventional therapy combined with Xinmailong injection on chronic heart failure," Guide of China Medicine, vol. 14, no. 20, pp. 10-12, 2016.

[25] Z. Han and Y. Liu, "Clinical observation of Xinmailong injection in the treatment of 56 cases of senile chronic heart failure with slow arrhythmia," China Pharmaceuticals, vol. 27, no. 05 , pp. 55-57, 2018.

[26] B. H. He, "Effect of furosemide and Xinmailong injection on left ventricular ejection fraction and blood lactic acid in patients with heart failure," Chinese Journal of Cardiovascular Rehabilitation Medicine, vol. 26, no. 05, pp. 529-533, 2017.

[27] H. B. Li and D. D. Li, "Clinical observation of Xinmailong injection in the treatment of chronic heart failure," Hebei Medical Journal, vol. 37, no. 05, pp. 713-714, 2015.

[28] Q. B. Li, "Clinical effect of Xinmailong injection on 48 cases of chronic heart failure," Journal of Gannan Medical University, vol. 36, no. 03, pp. 419-420, 2016.

[29] Z. G. Li, G. F. Ma, J. Yang, Q. Q. Zhang, and S. X. Geng, "Effect of Xinmailong injection combined with bisoprolol on hemodynamics and serum hs-CRP, BNP and VEGF in patients with coronary heart disease and heart failure," Chinese Journal of Integrative Medicine on Cardio-Cerebrovascular Disease, vol. 16, no. 4, pp. 393-397, 2018.

[30] H. L. Liu, R. B. Zhang, and H. Y. Wang, "Effect of Xinmailong on cardiac function and exercise tolerance in patients with ischemic cardiomyopathy and heart failure," The Journal of Medical Theory and Practice, vol. 31, no. 15, pp. 2209-2211, 2018.

[31] Q. Liu, "Clinical observation of Xinmailong combined with atorvastatin in the treatment of chronic heart failure," Acta Medicinae Sinica, vol. 31, no. 05, pp. 96-99, 2018.

[32] Y. H. Liu, X. . 1. Gao, X. K. Zhang, M. Xie, Y. N. Liu, and S. Q. Cui, "Effect of torasemide combined with Xinmailong injection on VEGF, BNP, NGAL and ICAM-1 in patients with chronic heart failure," Shaanxi Medical Journal, vol. 47, no. 10, pp. 1322-1324, 2018.

[33] W. Quan and Y. Miao, "Clinical observation of Xinmailong injection in the treatment of chronic heart failure," Chinese Journal of New Clinical Medicine, vol. 10, no. 04, pp. 353-356.

[34] W. Shen, Y. Li, and S. Yang, "Effect of Xinmailong injection on cardiac function and NT-proBNP in patients with coronary heart disease and heart failure," Chinese Journal of Integrative Medicine on Cardio-Cerebrovascular Disease, vol. 15, no. 07, pp. 833-835, 2017.

[35] H. R. Shi, Y. P. Feng, X. Q. Yang, J. W. Song, and X. Lu, "Effect of Xinmailong injection on BNP, hsCRP and VEGF in patients with coronary heart disease and heart failure," Chinese Journal of Integrative Medicine on Cardio-Cerebrovascular Disease, vol. 14, no. 02, pp. 168-170, 2017.

[36] M. Teng and X. Wang, "Clinical observation of Xinmailong injection combined with trimetazidine in the treatment of chronic heart failure of coronary heart disease (qiyang deficiency syndrome)," China Pharmacy, vol. 28, no. 26, pp. 3705-3707, 2017.
[37] L. Y. Wang, M. H. Hu, X. H. Zhang, L. W. Ma, and L. P. Ren, "Clinical observation on the improvement of heart function in patients with chronic heart failure of coronary heart disease," Chinese Journal of Integrative Medicine on Cardio-Cerebrovascular Disease, vol. 10, no. 11, p. 1380, 2012.

[38] Z. Wu, W. Chen, and X. Liu, "Effect of Xinmailong injection on serum BNP, hsCRP and VEGF in patients with coronary heart disease and heart failure," Chinese Archives of Traditional Chinese Medicine, vol. 35, no. 09, pp. 2433-2435, 2017.

[39] T. Wu and H. Yang, "Clinical effect of Xinmailong injection on ischemic cardiomyopathy with heart failure," Medical Journal of Chinese People's Health, vol. 27, no. 04, pp. 74-108, 2015.

[40] Y. Xu and F. Z. Xu, "Clinical effect of Xinmailong injection on chronic heart failure," Chinese Journal of Integrative Medicine on Cardio-Cerebrovascular Disease, vol. 14, no. 20, pp. 2413-2414, 2016.

[41] J. G. Xue, X. L. Wang, Y. Xu et al., “A multicenter randomized controlled study of Xinmailong injection in the treatment of chronic heart failure (Syndrome of deficiency of both qi and Yang and internal obstruction of blood stasis)," Chinese Journal of Integrated Traditional and Western Medicine, vol. 35, no. 07, pp. 796-800, 2015.

[42] J. Yang, G. H. Chen, B. Jiang, and X. Q. Yang, "Clinical observation of Xinmailong injection in 110 elderly patients with chronic heart failure," China Medical Herald, vol. 9, no. 14, pp. 93-99, 2012.

[43] N. Yang, H. Wang, and D. Si, "Clinical observation of Xinmailong injection combined with dopamine in the treatment of chronic heart failure," Drugs and Clinic, vol. 32, no. 05, pp. 796-799, 2017.

[44] G. L. Yuan, Y. Q. Zhao, X. T. Li, and M. Yang, "Clinical observation of Xinmailong injection in the treatment of chronic heart failure," Hebei Journal of Traditional Chinese Medicine, vol. 37, no. 10, pp. 1545-1548, 2015.

[45] C. M. Zhao, X. Z. Wang, Y. H. Guo, X. M. Zhang, and H. J. Li, "Effect of Xinmailong on serum MMP-9 in patients with heart failure," Chinese Journal of Gerontology, vol. 17, no. 02, pp. 173-176, 2014.

[46] Z. Y. Zhao, Z. Qi, X. E. Cui, F. An, and J. Wang, "Clinical study on the treatment of chronic heart failure with Xinmailong injection," Journal of Logistics University of PAP(Medical Sciences), vol. 19, no. 02, pp. 120-122, 2010.

[47] Y. Jiang, S. Li Bao, Y. P. Tang, M. Zhou, T. T. Li, and J. X. Chu, "Clinical study of Xinmailong injection in the treatment of chronic heart failure in the elderly," China Pharmaceuticals, vol. 28, no. 15, pp. 53-55, 2019.

[48] P. Z. Li, "Effects of Xinmailong injection combined with standard regimen on cardiac function and short-term prognosis in patients with chronic heart failure," Chinese Journal of Integrative Medicine on Cardio-Cerebrovascular Disease, vol. 17, no. 17, pp. 2618-2621, 2019.

[49] H. Y. Liu and Y. H. Zhou, "Effect of Xinmailong injection on chronic heart failure and its influence on NT-proBNP, hs CRP and PCT in serum," Chinese Journal of Gerontology, vol. 39, no. 15, pp. 3610-3613, 2019.

[50] L. Y. Zhang and Z. L. Zhou, "Effects of Xinmailong injection on cardiac function and vascular endothelial function in elderly patients with chronic heart failure," Journal of Qiqihar Medical University, vol. 40, no. 06, pp. 703-704, 2019.

[51] H. Li, Y. Mao, Q. Zhang et al., "Xinmailong mitigated epirubicin-induced cardiotoxicity via inhibiting autophagy," Journal of Ethnopharmacology, vol. 192, pp. 459-470, 2016. 
[52] Z. Li, S. Li, L. Hu et al., "Mechanisms underlying action of Xinmailong injection, a traditional Chinese medicine in cardiac function improvement," African Journal of Traditional, Complementary and Alternative Medicines, vol. 14, no. 2, pp. 241-252, 2017.

[53] Z. Q. Liu, H. B. Liu, and B. L. Wang, "Meta analysis of the safety of Xinmailong injection in the treatment of heart failure," China Pharmacy, vol. 29, no. 22, pp. 3152-3157, 2018.

[54] J. Wu, R. Nui, and C. Dong, "Effects of Xinmailong Xiangdan and Shenmai Injection on early kidney damage induced by toxin of grass carp bile," Zhong Xi Yi Jie He Xue Bao, vol. 2, no. 1, pp. 33-35, 2004.

[55] Q. J. Li, Z. G. Wang, Q. Liu, Y. Xie, and H. L. Hu, "Research status of Periplaneta americana with analyses and prospects of key issues," Zhongguo Zhong Yao Za Zhi, vol. 43, no. 7, pp. 1507-1516, 2018

[56] H. Riaz, S. U. Khan, H. Rahman et al., "Effects of high-density lipoprotein targeting treatments on cardiovascular outcomes: a systematic review and meta-analysis," European Journal of Preventive Cardiology, vol. 26, no. 5, pp. 533-543, 2019.

[57] H. Liu, Y. Yan, P. Pang et al., "Angong Niuhuang Pill as adjuvant therapy for treating acute cerebral infarction and intracerebral hemorrhage: a meta-analysis of randomized controlled trials," Journal of Ethnopharmacology, vol. 237, pp. 307-313, 2019.

[58] A. Walther, J. Breidenstein, and R. Miller, "Association of testosterone treatment with alleviation of depressive symptoms in men," JAMA Psychiatry, vol. 76, no. 1, pp. 31-40, 2018. 